\title{
ICD in the Era of Telecardiology
}

\author{
J. Taieb, J. Bouet, R. Morice, J. Hourdain, B. Jouve, \\ Y. Rahal, T. Benchaa, H. Khachab, O. Rica and \\ C. Barnay
}

Additional information is available at the end of the chapter

http://dx.doi.org/10.5772/53361

\section{Introduction}

Home monitoring of Pacemakers trans-telephonically was introduced in 1971 and remained until recently the main technology to remotely follow the performance of PMs. It was mostly aimed at ascertaining the integrity of the system especially with regard to battery performance and longevity, appropriate capture, and sensing.

Modern Remote wireless communication from the Cardiac implantable electronic devices (CIED) to a home communicator allows the transmission of the information gathered by the device regarding programming, test and alerts, to the clinician. It became the new standard for remote follow-up. [1-3]

The current CIEDs being interrogated remotely include implantable cardioverter defibrillator (ICDs), pacemakers, implantable loop recorders and implantable haemodynamic monitors. [2]

\section{Definitions and principles of telecardiology applicable to ICDs}

Telecardiology or home monitoring of ICDs refers to remote communication technology in general. Different types of data transmission are available.

\subsection{Type of transmissions}

Remote follow-up refers to programmable scheduled transmissions in which routine CIED parameters are collected remotely in a format similar to that obtained during a routine clinic visit. As opposed to trans-telephonically monitoring, practically all information available 
during traditional ICD interrogation with a programmer can be obtained via remote followup for a better outcome of patients [4-5]

Remote monitoring is an alert function. It refers to data acquired automatically with unscheduled transmissions of any pre-specified alerts related to device functioning or to clinical events. The latter adds a new functionality to implanted devices, opening a new era of potentially beneficial pre-emptive interventions that may alter the natural history of a particular disease or condition. [6-7].

Patient-initiated interrogations refers to non-scheduled follow-up interrogations as a result of a patient experiencing a real or perceived clinical event, for which the patient is seeking expert evaluation.

\subsection{Technology of transmission}

Unlike traditional follow up that implies a clinic appointment, transportation and face to face meeting, remote follow up is based on Data transmitted from the device to the home monitoring station by wireless communication or using a telemetry head (for older models) between device and home monitoring station. This home monitor is linked by telephone (analogic line or GSM ) to a central server or website automatically to deposit encrypted data for further analysis (figure 1).

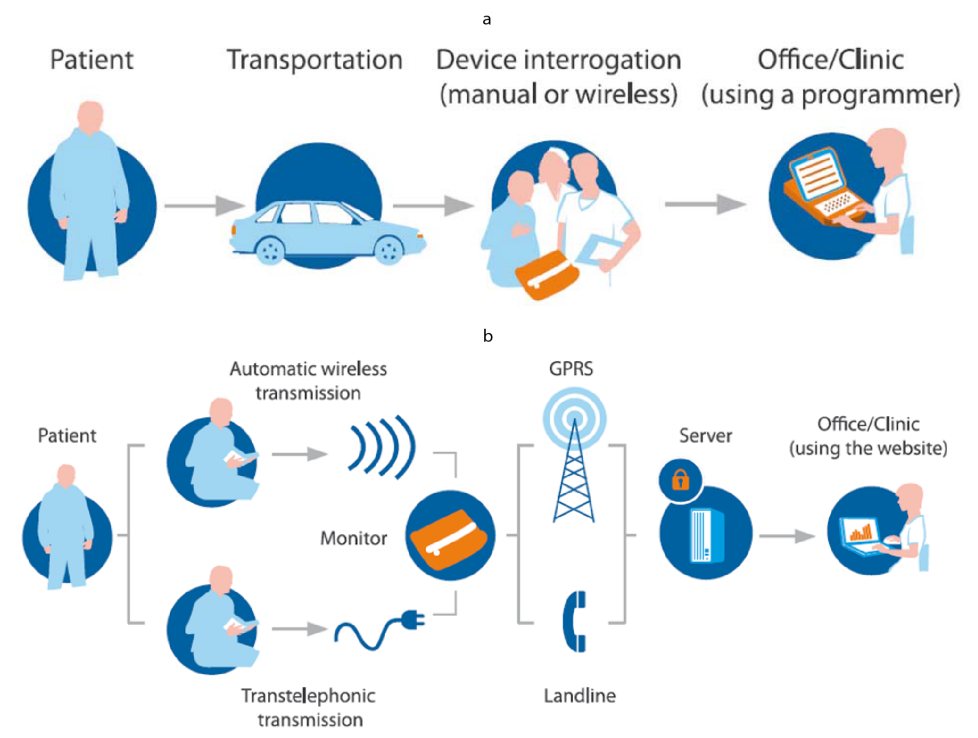

Figure 1. In clinic follow up (a) needs scheduling and transportation while Remote communication (b) is performed by a radiofrequency transmitter circuitry integrated in the ICD utilizing telephone lines or cellular phone technology. The ICD transfers encrypted data via the Transmitter to a service centre using a cellular network. The service centre provides a cardiologic report accessible online by the physician via a secure Internet access 
Alert notifications are sent to physicians via pager, fax, SMS, voice message, or email. Many systems require access to a dedicated (device or company specific) website to obtain detailed information on the interrogation.

Remote reprogramming of alert level (yellow or red) is possible but remote ICD programming is not yet available in clinical practice, mainly due to safety considerations regarding data protection and unauthorized control of device function.

ICD compatible with home monitoring use radiofrequency telemetry to send information to a home communicator. This feature allows also the device to be operated remotely on a short distance ( 3 to 7 meters) during implantation procedure hence more flexibility, shorter intervention time, and lower risk of infection.[8] Radiofrequency telemetry is also very useful during in person follow up, eliminating the need for patient preparation and ECG monitoring in most cases, all information being accessible via the programmer (figure 2). Unfortunately, this possibility of short distance remote ICD interrogation and programming is not fully available for all brands at this time. (table 1)

\begin{tabular}{|c|c|c|c|c|c|}
\hline & Boston & Biotronik Home & Medtronic & St Jude Merlin.net & Sorin SMARTVIEW \\
\hline & Latitude & & & & \\
\hline Network & $\begin{array}{l}\text { Analogue } \\
\text { phone line. }\end{array}$ & $\begin{array}{l}\text { GSM network } \\
\text { (Europe \& USA), } \\
\text { Analogue phone } \\
\text { line (USA } \\
\text { \&Japan) } \\
\text { 3G (Japan) }\end{array}$ & $\begin{array}{l}\text { Analogue phone } \\
\text { line and GSM } \\
\text { network }\end{array}$ & $\begin{array}{l}\text { Analogue phone } \\
\text { line, cellular } \\
\text { network and WiFi }\end{array}$ & $\begin{array}{l}\text { Analogue phone line } \\
\text { and GSM network }\end{array}$ \\
\hline $\begin{array}{l}\text { Physician } \\
\text { notification }\end{array}$ & $\begin{array}{l}\text { Fax, phone, } \\
\text { email }\end{array}$ & SMS, email, fax & SMS, email & $\begin{array}{l}\text { Fax, email, SMS } \\
\text { and automated } \\
\text { phone calls }\end{array}$ & Fax, email, SMS \\
\hline $\begin{array}{l}\text { IEGM (real-time at } \\
\text { remote follow-up) }\end{array}$ & $10 \mathrm{~s}$ & $30 \mathrm{~s}$ & $10 \mathrm{~s}$ & $30 \mathrm{~s}$ & $7 \mathrm{~s}$ \\
\hline $\begin{array}{l}\text { FDA and CE Mark } \\
\text { system approval }\end{array}$ & Yes & Yes & Yes & Yes & $\begin{array}{l}\text { CE yes } \\
\text { FDA (in progress) }\end{array}$ \\
\hline $\begin{array}{l}\text { Short distance RF } \\
\text { remote } \\
\text { programming }\end{array}$ & Yes & $\begin{array}{l}\text { Yes } \\
\text { (available in } \\
\text { 2012) }\end{array}$ & Yes & $\begin{array}{l}\text { Yes with an } \\
\text { antenna }\end{array}$ & no \\
\hline
\end{tabular}

Table 1. Comparaison of communications features 
a

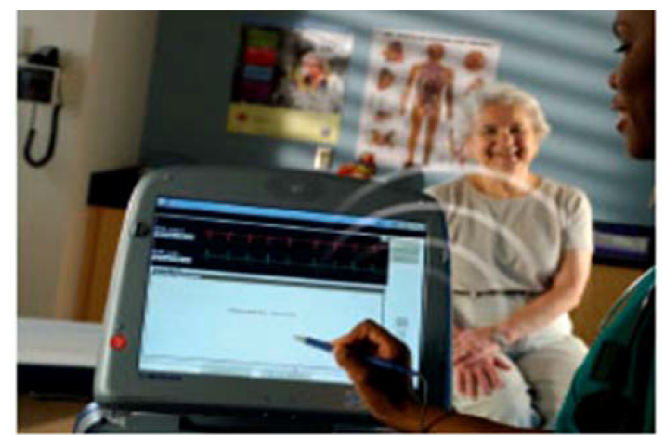

b

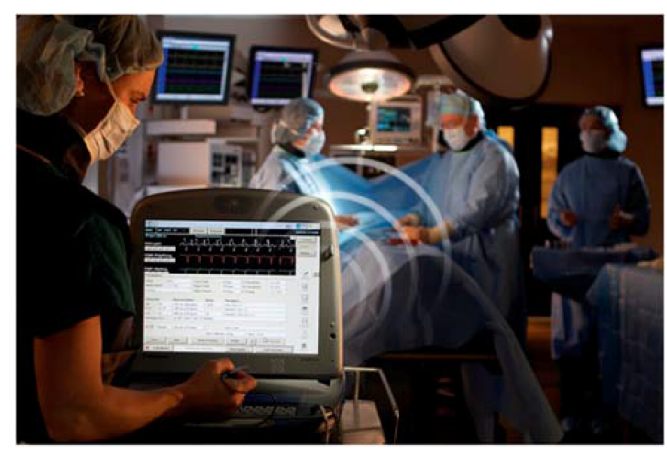

Figure 2. Some manufacturers use RF to interrogate and program remotely on a short distance. This is of particular interest during in person follow up (a) and during implantation procedure (b)

\subsection{Types of alerts}

The ability of implantable devices to continuously monitor variables such as heart rate, $[9,10]$ the patient's daily activities, [11] intrathoracic impedance for the detection of fluid accumulation, [12] the occurrence of arrhythmias [13,14] and the integrity of the system [15] may provide early warning of changes in cardiac status or of safety issues and allow timely management. When these patients have clinical events such as ICD shocks or device audible alert notifications of possible critical situations, they often visit the emergency department or clinic for an unscheduled examination.

ICD and lead dysfunction may be associated with severe consequences and could be anticipated thanks to home monitoring alerts. Patients could be contacted to correct the problem in office for reprogramming or in hospital if a procedure is needed. [16-17]

Significant change in lead impedance, pacing or sensing thresholds, could be linked to lead failure and should be investigated thoroughly. It has been reported that remote monitoring helps prevent inappropriate shocks in a population at risk. [18] 
An hemodynamic measurement modification, a low rate of resynchronisation should lead physicians to look for an aetiology (e.g. atrial fibrillation, Av delay, crosstalk...) in order to avoid a cardiac heart failure.

Development of persistent atrial fibrillation with fast ventricular rate close to ventricular fibrillation (VF) zone, frequent episodes of ventricular tachycardia (VT) with delivery of frequent antitachycardiac pacing sequences, should also act as a trigger for a follow up visit in order to change ICD parameters (VT, VF Zones, discrimination algorithms), or drug therapy.

\subsection{Home monitor communicator}

This is a remote telemetry device able to communicate with the ICD automatically in real time or at scheduled intervals, and that transmits the encrypted data over long distances utilizing telephone lines or cellular phone technology. The data are then entered and stored in dedicated servers that act as data repositories and communicate actively or passively with the caregivers of the patient. A specific home communicator was developed by each company: Medtronic Care- Link, Boston Scientific Latitude, Biotronik Home Monitoring, Sorin Smart View and St Jude Merlin@Home.net. In the near future, all systems will be compatible with GSM. Besides, Biotronik offers complete mobility of the home monitoring station with battery backup. Furthermore, frequency of remote follow up, and selection of remote monitoring alerts are fully programmable in all systems

\section{Advantages and challenges of telecardiology}

\subsection{Physicians}

Because of the burden of follow-up of ICD patients, with regular in-office visits every 3-6 months, puts on specialized electrophysiology clinics Heidbuchel et al. [19] retrospectively evaluated in 1739 ICD visits in a random set of 169 patients. The standard follow-up scheme consisted of in office visits 1 month after implantation and then every 6 months, unless approaching battery depletion. They conclude that ICD remote monitoring can potentially diagnose $99.5 \%$ of arrhythmia or device-related problems if combined with a follow-up by the local general practitioner and/or referring cardiologist. Its use may provide a way to significantly reduce in-office follow-up visits that are a burden for both hospitals and patients. A similar study was performed by Elsner et al. [20]. They investigated in a prospective, randomized, and multicentre comparison study the effect of ICD home monitoring against conventional follow-up in 115 MADIT II patients. The results prove that the simplified ICD follow-up scheme with additional home monitoring in MADIT II patients can reduce the number of visits and lead to time reduction.

In 2011 Boriani et al. published a survey [21] indicating that in 'real-world' clinical practice, the follow-up of CIEDs requires important resources in terms of time dedicated by special- 
ized personnel, corresponding to cardiologists, nurses, internal technicians, and also, external, industry-employed technicians.

More recently Cronin et al. [22] found that analysis of remote monitoring transmissions has significant implications for device clinic workflow. Non-actionable transmissions are rapidly processed, allowing clinicians to focus on clinically important findings.

According to Theuns et al. [24]: "remote monitoring is feasible, may facilitate ICD follow up, and lead to early detection of system-related complications. Continuous monitoring of specific device parameters may avoid unnecessary replacements of devices or leads. However, as with every new technology, there are areas of uncertainty. Remote monitoring is associated with a redesigned organization of the care system, including physicians, allied professionals, and a dedicated remote monitoring service. Another area of uncertainty is related to the question of liability. The now "virtual patient" poses a paradigm shift. Physicians have the responsibility for responding to the new sources of data. How fast must a physician react to the transmitted alerts? Do we need 24 hours, 7 days a week coverage or is it legally acceptable not to check event notifications outside the office hours ? The development of practice guidelines on the appropriate role of remote monitoring of patients with implanted cardiac devices would help to address many of these issues."

\subsection{Patients}

Besides the decrease in number of in office follow up, safety and more rapid detection of actionable events compared with conventional monitoring in patients with implantable electronic cardiac devices were demonstrated in several studies:

In Lumos-T Safely Reduces Routine Office Device Follow-up (TRUST) multicentre trial [27, 28] authors concluded that home monitoring detected more device related issues and earlier compared with those following calendar-based or symptom-driven in-person interrogations. The results confirmed that conventional in-person follow-up methods underreport device malfunctions.

In the AWARE Study [29], Lazarus et al. analysed transmissions of 11624 recipients: 4631 pacemakers, 6548 single or dual chamber defibrillators and 445 cardiac resynchronisation therapy defibrillators (CRT-D) systems. The mean interval between the last follow-up and the occurrence of events notified by home monitoring was 26 days, representing a putative temporal gain of 154 and 64 days in patients usually followed up at 6 and 3 month intervals, respectively.

In 2010, the ALTITUDE registry showed that for the 69556 ICD and CRT-D patients receiving remote follow-up on the network, 1 and 5 year survival rates were higher compared with those in the 116222 patients who received device follow-up in device clinics only (50\% reduction; $p=0.0001$ ) [30].

Another example of remote monitoring improving clinical outcomes is its potential to reduce symptomatic lead failures, consisting of inappropriate shocks and symptomatic pacing inhibition due to oversensing. A study of patients who underwent repeat surgery due to 
malfunctions of the ICD lead compared the rate of symptomatic lead failure in patients monitored remotely with those followed up in-clinic [31]. Inappropriate shocks occurred in $27.3 \%$ of the remote group compared with $46.5 \%$ of the in-clinic group. This trend gains statistical significance if the compound endpoint of inappropriate shocks and symptomatic pacing inhibition due to oversensing is focused; $27.3 \%$ in the remote group compared with $53.4 \%$ in the in-clinic group. The remote monitoring system sent alert messages in $91 \%$ of all incidents, enabling intervention to prevent aninappropriate shock.

Mabo reported in the EVATEL study [32], a randomized trial that included 1500 patients implanted with single or dual chamber ICD that "Home monitoring leads to a decrease of $37 \%$ of inappropriate shocks.

Kacet reported similar results in the ECOST study [33]: "home monitoring reduces by $76 \%$ the number of aborted ICD charges with a significant impact on battery status and device longevity".

Raatikainen et al. [34] reported that over $90 \%$ of patients found the system easy to use. Marzegalli et al. [35] also reported that the review procedure was successful. Its mean duration was $5 \pm 2$ minutes per transmission and users indicated that both access and navigation were easy. Patients reported a general preference for remote versus in clinic follow-up and described a sense of reassurance created by the remote monitoring capacity. In a study of 379 patients implanted with pacemakers, Halimi et al. reported all differences in the SF-36 questionnaire scores to be non-statistically significant [36]. Patient satisfaction was studied recently by Petersen et al. [23]: of the 385 of the patients that answered the survey $(81.2 \%)$, ninety-five percent were content with the remote Follow up. Only $25 \%$ had unscheduled transmissions and most unscheduled transmissions were for appropriate reasons. Eightyfour percent of the patients wished for a more detailed response and $21 \%$ wished for a faster reply after routine transmissions.

Current ICDs provide not only arrhythmia information but also several indicators of heart failure (HF). Studies are under way to evaluate the benefits of HF specifics diagnostics coupled with home monitoring $[25,26]$.

\subsection{Health economics}

While remote monitoring ( $\mathrm{RM}$ ) may be able to reduce the time spent on device follow up it is not clear whether this relates to an overall reduction in costs. RM has its own costs including the cost of the transmitter, the setup and maintenance of the central server and database, patient and clinic staff education and staff time to read, interpret, import information into electronic medical records and act on transmission events/problems. The frequency of in office visits and the frequency of RM transmissions, proximity of the patient to the clinic and many other factors will affect the economic modeling as to the potential cost savings associated with RM. Beyond assessing the simple economic modeling is the assessment of cost effectiveness which also needs to consider the improvement in patient outcomes as well as the costs involved for each form of follow up. 


\subsection{Limits of telecardiology}

A network failure may delay transfer of data. Most of telecardiology departments do not have 24/7services. Thus, an alert message issued on a Friday night has a good chance not to be examined before the following Monday. In addition transferred data through the network are privileged, leading to legal considerations regarding reliability of the technology and confidentiality especially during emergency situations. To add another level of complexity, each country seems to have a different modus operandi at this point in time.

Health care providers and health care organizations that are involved in remote monitoring (RM) of ICDs will typically sign a 'Terms of Use' agreement with each of the ICD vendors. These legal documents outline the provisions of RM between the ICD vendor and the user. The patient needs to be informed of the purpose and limitations of RM, such as the fact that it does not replace an emergency service or absence of dealing with alert events outside office hours. Before initiating RM and follow-up, the patient may be requested to sign a written informed consent stating these points and authorizing transmission of personal data to third parties, respect of privacy, and confidentiality of patient data by device companies should be subjected to strict rules, described in contracts. Cardiac implantable devices record a wealth of information and as devices become more sophisticated the scope of information can be expected to grow. Guidelines need to be established to determine the periodicity with which ICD transmissions would need to be reviewed and documented.

Vulnerability of security breaches by hackers accessing devices with wireless capability must be tested in every system. There have been no reports to date of unauthorized reprogramming of implantable devices; however, unauthorized access to personal information stored on internet servers must be also considered.

In addition, transfer of ICD data would be impossible if the home monitoring station is not close to the patient at reasonable time intervals. This could be happening in case of hospitalization in another center. The patient could even experience serious system failure without any data transmission.

Logistics may also be a limit to the development of home monitoring: It is up to the implanting center to organize ordering, stock management and traceability of home monitoring stations as well as patient education. The Sorin group is the only one so far using a distribution network to handle all these tasks.

\subsection{Reimbursement}

Reimbursement is important to the manufacturer in order to compensate for some of the costs related to the home monitoring stations and the transmission network. It remains a major concern in most countries, limiting the increase of use of remote monitoring despite growing evidence in favor of this technology. Today's cost containment pressure requires increased reimbursement efforts with the burden of proof shifting to medical communities and manufacturers. Reimbursement assessments often begin with the presumption that a technology or service will not be covered unless its use is supported by scientific evidence of improved outcomes. Recent publications like the EVOLVO study [37] are important milestones in this en- 
deavor. It concludes that "remote monitoring can reduce emergency department/urgent in office visits and, in general, total healthcare use in heart failure patients with modern ICD/ CRT-D. Compared with standard follow-up through in-office visits and audible ICD alerts, remote monitoring results in increased efficiency for healthcare providers and improved quality of care for patients". Another study is under way to develop a cost minimization analysis from the hospital perspective and a cost effectiveness analysis from the third payer standpoint, based on direct estimates of costs and QOL associated with remote follow-ups, compared with standard ambulatory follow-ups, in the management of ICD and CRT-D recipients [38].

\section{Conclusion}

Remote monitoring of ICDs represents a growing area with increasing numbers of patients being subject to these technologies but also more and more physicians involved in decision making on the indications for these technologies and the handling of data in the context of clinical decision making.

Cardiac implantable device transmissions may occur either over telephone lines or over cellular network lines. These transmissions often only take less than a minute to a few minutes to complete. However, in the foreseeable future we can expect alternative methods of data transmission to become available with transmission rates that will make it possible for nearly continuous and instantaneous patient ICD data delivered to health care providers. There are, of course, limitations to how frequently ICD data can be reviewed by health care providers and battery longevity constraints will likely limit the transmission times as well.

Technological advancements continue to structure our practice of medicine, but with it often new legal challenges emerge. In order to minimize risk to patient and liability to health care providers a clear discussion regarding the expectations and limitations of remote monitoring between patients and health care providers is recommended

\section{Acknowledgements}

The authors thank Muriel Bon, Michelle Hartenstein, Xavier Laroche, Ariane Szczygiel, Marion De Matteis, Moti Daswani and Vincent Desplat for their expert technical assistance, and Fabrice Chomienne, for his help in the composition of the manuscript.

\section{Author details}

J. Taieb, J. Bouet, R. Morice, J. Hourdain, B. Jouve, Y. Rahal, T. Benchaa, H. Khachab, O. Rica and C. Barnay

Hospital Center of Aix en Provence, France 


\section{References}

[1] Wilkoff BL, Auricchio A, Brugada J et al. HRS/

EHRA expert consensus on the monitoring of Cardiovascular Implantable Electronic Devices (CIEDs). Europace 2008;10:707-25.

[2] Dubner S, Auricchio A, Steinberg JS, et al SHNE/EHRA expert consensus on remote monitoring of cardiovascular implantable electronic devices (CIEDs) Annals of Noninvasive Electrocardiology 2012;17:35-36

[3] Winters SL, Packer DL, Marchlinski FE et al. North American Society of Electrophysiology and Pacing. Consensus statement on indications, guidelines for use, and recommendations for follow-up of implantable cardioverter-defibrillators. Pacing Clin Electrophysiol 2001;24:262 - 9 .

[4] Crossley GH, Chen J, Choucair W, et al, on behalf of the PREFER Study Investigators. Clinical benefits of remote versus transtelephonic monitoring of implanted pacemakers. J Am Coll Cardiol 2009;54:2012-9.

[5] Crossley G, Boyle A, Vitense H, et al. The Clinical Evaluation of Remote Notification to Reduce Time to Clinical Decision (CONNECT) Trial. Am Heart J 2008;156:840-6

[6] Joseph GK, Wilkoff BL, Dresing T, et al. Remote interrogation and monitoring of implantable cardioverter defibrillators. J Interv Card Electrophysiol 2004;11:161 - 6

[7] Ricci RP, Morichelli L, Santini M. Home monitoring remote control of pacemaker and ICD patients in clinical practice. Impact on medical management and health care resource utilization. Europace 2008;10:164-70.

[8] Schoenfeld MH, Reynolds DW. Sophisticated remote implantable cardioverter defibrillator follow-up: a status report. Pacing Clin Electrophysiol 2005;28:235-40.

[9] Adamson PB, Smith AL, Abraham WT, et al; InSync III Model 8042 and Attain OTW Lead Model 4193 Clinical Trial Investigators. Continuous autonomic assessment in patients with symptomatic heart failure: prognostic value of heart rate variability measured by an implanted cardiac resynchronization device. Circulation. 2004;110:2389 -2394.

[10] Landolina M, Gasparini M, Lunati M, et al; InSync/InSync ICD Italian Registry Investigators. Heart rate variability monitored by the implanted device predicts response to CRT and long-term clinical outcome in patients with advanced heart failure. Eur J Heart Fail. 2008;10:1073-1079.

[11] Kadhiresan VA, Pastore J, Auricchio A et al; PATH-CHF Study Group. Pacing therapies in congestive heart failure: a novel method - the activity log index-for monitoring physical activity of patients with heart failure. Am J Cardiol. 2002;89: 1435-1437. 
[12] Yu CM, Wang L, Chau E, et al. Intrathoracic impedance monitoring in patients with heart failure: correlation with fluid status and feasibility of early warning preceding hospitalization. Circulation. 2005;112:841-848.

[13] Capucci A, Santini M, Padeletti L, Gulizia M et al; Italian AT500 Registry Investigators. Monitored atrial fibrillation duration predicts arterial embolic events in patients suffering from bradycardia and atrial fibrillation implanted with antitachycardia pacemakers. J Am Coll Cardiol. 2005;46:1913-1920.

[14] Santini M, Gasparini M, Landolina M, et al cardiological centers participating in Clinica 1Service Project. Device detected atrial tachyarrhythmias predict adverse outcome in real-world patients with implantable biventricular defibrillators. J Am Coll Cardiol. 2011;57:167-172.

[15] Gunderson BD, Patel AS, Bounds CA, et al. An algorithm to predict implantable cardioverter defibrillator lead failure. J Am Coll Cardiol. 2004;44:1898 -1902

[16] Joseph GK, Wilkoff BL, Dresing T, et al. Remote interrogation and monitoring of implantable cardioverter defibrillators. J Interv Card Electrophysiol 2004;11:161-6.

[17] Ricci RP, Morichelli L, Santini M. Home monitoring remote control of pacemaker and ICD patients in clinical practice. Impact on medical management and healthcare resource utilization. Europace 2008;10:164-70.

[18] Banck Z, Axtell K, , Brodhagen K, et al. Inappropriate Shocks in Patients With Fidelis ${ }^{\circledR}$ Lead Fractures: Impact of Remote Monitoring and the Lead Integrity Algorithm Journal of Cardiovasc Electrophysiol, 2011;22, :1107-1114,

[19] Heidbuchel H, Lioen P, Foulon S et al. Potential role of remote monitoring for scheduled and unscheduled evaluations patients with an implantable defibrillator. Europace 2008;10:351-7.

[20] Elsner C, Sommer P, Piorkowski C, et al. A Prospective Multicenter Comparison Trial of home monitoring against regular follow-up in MADIT II. Comput Cardiol 2006;33:241-4.

[21] Boriani G, Auricchio A, Klersy C, et al. Healthcare personnel resource burden related to in-clinic follow-up of cardiovascular implantable electronic devices: a European Heart Rhythm Association and Eucomed joint survey. Europace $2011 ; 13$ :1166-1173

[22] Cronin, E.M., Ching, E.A., Varma, N. et al, Remote monitoring of cardiovascular devices - a time and activity analysis, Heart Rhythm 2012, doi: 10.1016/j.hrthm. 2012.08.002.

[23] Helen H Petersen, Mie C Jensen Larsen, Olav W Nielsen, et al. Patient satisfaction and suggestions for improvement of remote ICD monitoring. J Interv Card Electrophysiol 2012 34:317-324 
[24] Theuns D. A. M. J. ,Jordaens L. Use of remote monitoring in the management of system-related complications in implantable defibrillator patients. Neth Heart J 2012 20:82-85

[25] Brachmann J, 1 Bohm M, Rybak K, et al on behalf of the OptiLink HF Study Executive Board and Investigators. Fluid status monitoring with a wireless network to reduce cardiovascular-related hospitalizations and mortality in heart failure: rationale and design of the OptiLink HF Study (Optimization of Heart Failure Management using OptiVol Fluid Status Monitoring and CareLink). European Journal of Heart Failure 2011;13:796-804

[26] Sack S, Wende CM, Nagel H et al . Potential value of automated daily screening of cardiac resynchronization therapy defibrillator diagnostics for prediction of major cardiovascular events: results from Home-CARE (Home Monitoring in Cardiac Resynchronization Therapy) study European Journal of Heart Failure 2011;13:10191027

[27] Varma N, Michalski J, Epstein AE, et al. Automatic remote monitoring of ICD lead and generator performance: the TRUST trial. Circ Arrhythm Electrophysiol 2010;3:428-36.

[28] Varma N, Epstein A, Irimpen A, et al. The lumos-T safely reduces routine efficacy and safety of automatic remote monitoring for implantable cardioverter-defibrillator follow-up. The Lumos-T Safely Reduces Routine Office Device Follow-Up (TRUST) Trial. Circulation 2010;122:325-32.

[29] Lazarus A. Remote, wireless, ambulatory monitoring of implantable pacemakers, cardioverter defibrillators, and cardiac resynchronization therapy systems: analysis of a worldwide database. Pacing ClinElectrophysiol 2007;30(Suppl 1):S2-12.

[30] Saxon LA, Hayes DL, Gilliam FR et al. Long-term outcome after ICD and CRT implantation and influence of remote device follow-up: the ALTITUDE survival study. Circulation 2010;122:2359-67. Epub 2010 Nov 22.

[31] Spencker S, Coban N, Koch L, et al. A Potential role of home monitoring to reduce inappropriate shocks in implantable cardioverter defibrillator patients due to lead failure. Europace 2009;11:483-8.

[32] Mabo P. Remote follow-up of patients implanted with an ICD. The prospective randomized EVATEL study. European Society of Cardiology 2011. Hotline 2. 29 août 2011.

[33] Kacet S. Safety of implantable cardioverter defibrillator follow-up using remote monitoring : a randomized controlled trial. Session Hot Line 2. Congrès de l'European Society of Cardiology, Paris, 29 août 2011

[34] Raatikainen MJP et al. Remote monitoring of implantable cardioverter defibrillator patients: a safe, time-saving, and cost-effective means for follow-up Europace 2008;10(10):1145-51 
[35] Marzegalli M et al. Remote Monitoring of CRT-ICD: The Multicenter Italian CareLink Evaluation-Ease of Use, Acceptance, and Organizational Implications PACE 2008;31:1259-64

[36] Halimi F, Clementy J, Attuel P, et al, on behalf of the OEDIPE trial Investigators. Optimized post-operative surveillance of permanent pacemakers by home monitoring: the OEDIPE trial. Europace 2008;10:1392-9.

[37] Landolina M, ; Perego GB , Lunati M,et al. Remote Monitoring Reduces Healthcare Use andImproves Quality of Care in Heart Failure Patients WithImplantable DefibrillatorsThe Evolution of Management Strategies of Heart Failure Patients With Implantable Defibrillators (EVOLVO) Study. Circulation. 2012;125:2985-2992

[38] Ricci RP , D'Onofrio A, Padeletti L, et al. Rationale and design of the health economics evaluation registry for remote follow-up: TARIFF. Europace doi:10.1093/europace/eus093 in press 
